\title{
Comparison of Salivary Cotinine Levels of Neonates Born to Mothers Exposed to Varying Degrees of Passive Cigarette Smoke
}

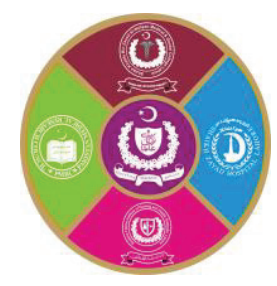

${ }^{1}$ Wardah Anwar, ${ }^{1}$ Ambreen Anjum, ${ }^{2}$ Nasreen Akhtar, ${ }^{3}$ Maria Anwar, ${ }^{4}$ Tabinda Kazmi, ${ }^{5}$ Sibgha Zulfiqar, ${ }^{6}$ Faiza Khan

${ }^{1}$ Department of Physiology, Al-Aleem Medical College, Lahore

${ }^{2}$ Department of Forensic Medicine \& Toxicology, Al-Aleem Medical College, Lahore

${ }^{3}$ Department of Physiology, Independent Medical College, Faisalabad

${ }^{4}$ Department of Physiology, Niazi Medical and Dental College, Sargodha

${ }^{5}$ Department of Physiology, Shaikh Zayed Medical Complex, Lahore

${ }^{6}$ Department of Pharmacology, Al-Aleem Medical College, Lahore

\begin{abstract}
Introduction: Obstetrical data reveals that fetuses born to mothers who are exposed to passive smoking show adverse health outcomes. Aims \& Objectives: To compare the cotinine levels in neonates born to mothers exposed to varying degrees of passive cigarette smoke versus the control group i.e., mothers who are not exposed to smoking. Place and duration of study: This study was conducted in Obstetrics and Gynecology department of Shaikh Zayed Hospital, Lahore and in emergency Labor Room of Ganga Ram hospital, Lahore from December 2015 to May 2016. Material \& Methods: This was a cross-sectional comparative study in which neonatal salivary cotinine level were measured and its relationship with birth weight was observed in 120 subjects, divided in four groups: i) neonates whose mothers were not-exposed to smoke, neonates whose mothers were exposed to, ii) 1-5 cigarette, iii) 6-10 cigarettes and iv) more than 10 cigarettes per day. Results: Salivary cotinine levels were raised in all four groups with maximum levels seen in group four but there was no statistical difference between groups. Changes in the birth weight were also seen with increased exposure to passive smoking. Conclusion: Salivary cotinine concentration in neonates increases due to passive exposure to cigarette smoke in mothers because of disregard of smokers to the adverse effects of nicotine inside homes and almost no governmental enforcement of laws regarding cigarette smoking in public spaces.
\end{abstract}

Key words: Passive smoking, salivary cotinine, neonate, non invasive

\section{INTRODUCTION}

$\mathrm{C}$ otinine is derived from breakdown of nicotine. $70-80 \%$ of nicotine is converted to cotinine in humans. This conversion occurs in two steps. In the first step cytochrome $\mathrm{P} 450$ system produce nicotine$\Delta 1^{\prime}\left(5^{\prime}\right)$-iminium and 5'-hydroxynicotine. ${ }^{1}$ In the second step cytoplasmic aldehyde oxidase acts as a catalyst to produce cotinine. , $^{2,4}$

Cotinine is measured in blood, hair, urine and saliva. ${ }^{5,6,7,8}$ Its measurement is mostly preferred because of their noninvasive nature as compared to blood-derived assays. ${ }^{9,10}$ Out of aforementioned techniques, saliva from neonates within 24 hours of birth has not been explored among noninvasive method in Pakistan. Most studies have found increasing level of Cotinine with increasing level of self-reported exposure to tobacco smoke. $^{11}$
However, not enough focus has been given to Second Hand Smoking (SHS) exposure to the neonate health. Our study combines the quantitative Nicotine exposure through Cotinine level with the questionnaire feedback.

Measuring Cotinine is preferred to measuring nicotine because nicotine has half-life of 2 hours whereas Cotinine remains in the body longer and is preferred due to its longer half-life of 17 hours. ${ }^{12,13,14}$

In humans birth weight is affected by the development during intrauterine period. ${ }^{15}$ Birth weight is very important and significant as it is associated with likelihood of mortality and morbidity.

According to a report of UNICEF, neonatal birth weight is good indicator of mother's health but also indicates the chances of survival of the baby. ${ }^{16}$ 
At term that is $37+$ weeks, normal birth weight should be $2500-4200$ grams ( 5 lbs. 8 ounces till 9 lbs. 4 ounces).

1) Birth weight less than $2500 \mathrm{~g}$, is low birth weight.

2) Birth weight less than $1500 \mathrm{~g}$, is known as very low birth weight.

3) Birth weight less than $1000 \mathrm{~g}$, is extremely low birth weight.

Out of these almost 2/3rd of the low birth weight infants are due to preterm birth and almost $1 / 3$ rd of these have growth restriction during intrauterine life. ${ }^{17}$

It has been well known that active smoking by mother is one of the environmental factor can decrease the birth weight of the infant markedly. There is an increase incidence of low birth weight infants born to smokers than to non-smokers. ${ }^{18,19}$ Passive maternal smoking increases the likelihood of lowering the birth weight of new born. Other environmental factors are

a) Lead Poisoning

b) Combustion Products of Solid Fuel

c) Carbon Mono Oxide

d) Mercury

e) Noise

Early morphological changes of the placenta are induced by Cotinine, resulting in reduced volume of maternal intervillous space and also surface area of fetal capillaries. This leads to reduction in oxygen diffusion across the placenta. ${ }^{20}$ The fetus suffers from chronic hypoxic stress as an outcome of smoking. ${ }^{21}$ These factors contribute to a reduced birth weight and length, and a smaller head circumference at birth..$^{22,23}$

When normal placenta develops, its blood supply depends on the growth of maternal decidua and the fetal trophoblast. The trophoblastic cells undergo growth, maturation, migration and invade the decidual cells. If for any reason these migrations and penetration of cell is interrupted, it leads to less growth of fetus. It has been established that nicotine exposure has an endocrine like effect on the development of the placenta. ${ }^{24}$

Exposure of nicotine obstructs the delivery of oxygen by causing vasoconstriction of all the blood vessels of body especially blood vessels of the umbilical cord. Not only this but the red blood cells combine with car-boxyhemoglobin. ${ }^{25}$ The combination further reduces the oxygenation of tissues. As a result of decreased oxygenation of fetal tissues, the growth and development of baby is devastated. Exposure of nicotine and its metabolites also cause direct damage to genetic material of fetus with impairment of lung development as well as increasing the heart rate and a decrease in breathing movement of fetus. ${ }^{26,27,28}$ Efforts are being made to avoid the incidence of lower than normal birth weight but these are not fruitful yet. There are other factors that increase the risk of infant mortality, the most important of which are socio-economic factors, medical risks before or during gestation and maternal lifestyles. ${ }^{29}$

Keeping in view the potential damaging effects of nicotine exposure in mothers this study was conducted to compare the Cotinine level of infants born to mothers who were divided in different groups. i) Unexposed to cigarette, ii) exposed to 1-5 cigarettes, iii) 6-10 cigarettes and iv) more than 10 cigarettes per day.

\section{MATERIAL AND METHODS}

This study was conducted in Obstetrics and Gynecology department of Shaikh Zayed Hospital, Lahore and in emergency Labor Room of Ganga Ram hospital, Lahore, after approval from The Ethical Committee and permission from the respective head of department. The study was conducted from December 2015 to May 2016.

Full term neonates, born to mothers aged (20 to 35 years), of same socioeconomic status and resident of Lahore were included in the study. Control group was neonates whose mothers were unexposed to passive smoking. Other groups were of neonates born to mothers who were exposed to passive smoke. Neonates of mothers with hypertension, respiratory diseases, cardiovascular disease, HIV infection and complicated pregnancy like anemia, poor weight gain were excluded. Also the actively smoking mothers (Cigarette, Huqqa, Cigar, etc.) or addicts to drugs, Alcohol, Pan and Naswaar chewing were excluded.

The sample size of 120 neonates divided into four equal groups (30 participant in each) was estimated by using $95 \%$ confidence level, $90 \%$ power of test with expected average Cotinine levels (in Nano grams per milliliter) of $0.077,0.257,0.466$ and 1.013 for non-exposed, less than 05 cigarette per-day 6-10 per-day cigarette and more than 10 cigarette consumption respectively. ${ }^{30}$

A questionnaire was developed in order to collect information regarding the tobacco exposure in the study group. The questionnaire was selfadministered and answered by mothers at the time of saliva collection in the post-natal ward. Personal data including name, age, and weight at first visit was recorded from their antenatal cards.

Detailed history and examination was performed and the birth weight was recorded on study Performa used as study tool. 
Saliva was taken from 120 neonates which were divided into four groups of 30 each according to the amount of maternal exposure to smoking i.e. i) nonexposed, ii) exposed to less than 5 cigarette per day, iii) exposed to 6-10 cigarette per day and iv) exposed to more than 10 cigarette per day each.

The saliva of new born was collected within 24 hours of birth with sterile dropper. Samples were collected at least $10 \mathrm{~min}$ before feeding and stored in Eppendorf tubes. Samples were kept cold in refrigerator and frozen at $-20^{\circ} \mathrm{C}$ within 4 hours after collection, in order to avoid bacterial growth in the specimen. Test was done by Elisa Technique in immunoassay laboratory, NHRC, Lahore by Calbiotech Cotinine kit , a solid phase competitive ELISA.

Comparison between groups was made using one way ANOVA followed by applying Tukey's test where applicable. P-value of $\leq 0.05$ for Cotinine level and birth weight of neonate was considered significant statistically.

Statistical analysis:

Data was entered and analyzed by using SPSS version 20.0.

\section{RESULTS}

In this study, 120 pregnant females were enrolled and saliva from their neonates was tested. They were divided into four groups according to different levels of cigarette exposure. Group 1 was considered as control group having neonates born to mothers without passive smoke exposure. The neonates in Group 2 were of mothers who were exposed to less than 05 cigarettes per day. Group 3 had neonates whose mothers were exposed to 05 to 10 cigarettes per day. While Group 4 was of those neonates whose mothers had exposure to more than 10 cigarettes per day.

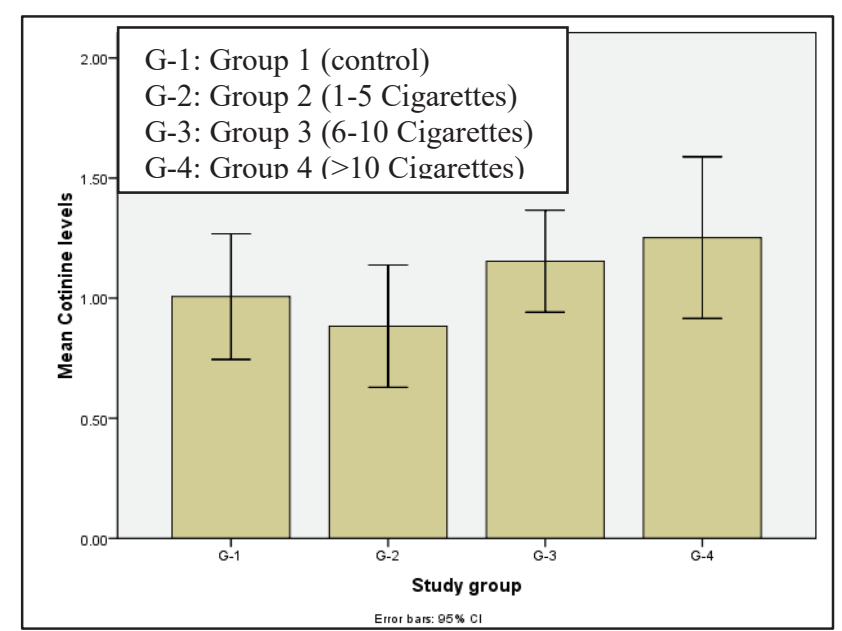

Fig-1: Mean Cotinine Level (ng/ml) of all four groups with standard deviation.
Mean cotinine levels in group- 1 was $1.0063 \pm 0.70$, in group-2 was $0.883 \pm 0.68$, in group-3 was 1.1537 \pm 0.57 and in group- 4 was $1.2520 \pm 0.90$. p-value of cotinine level was 0.216 which was not significant. Mean birth weight $(\mathrm{kg})$ in group-1 was $3.31 \pm 0.50$, in group-2 was $3.00 \pm 0.28$, in group-3 was $2.92 \pm$ 0.48 and in group- 4 was $2.66 \pm 0.41$. It was seen that birth weight was significantly different in all study groups as p-value was 0.001 . Mean birth weight of neonates showed inverse relation with SHS exposure of the mothers.

Our results show that in group 1 and group 4 there was positive but insignificant Pearson correlation between cotinine levels and birth weight that is $(\mathrm{r}=0.057, \mathrm{p}$-value $=0,767),(\mathrm{r}=0.046, \mathrm{p}$-value $=0.810)$ respectively. The Pearson correlation in groups 2 and 3 showed insignificant correlation, having $(\mathrm{r}=-$ $0.015, \mathrm{p}$-value $=0.936)(\mathrm{r}=-0.159, \mathrm{p}$-value $=0.402)$ respectively.

\section{DISCUSSION}

Mother and child are the two innocent members of our society, which suffer from the hazards of the passive/second hand smoke (SHS). Levels of SHS during pregnancy can be measured by newborn salivary cotinine levels. ${ }^{31}$ In our study we took the pregnant women of similar age and demographic characters, like all participants were residents of Lahore for last one year. We made a questionnaire in which the data of mother and the baby was recorded specially the history of exposure to second hand smoke and the birth weight.

We compared the maternal second hand exposure to cigarettes as reported in the questionnaire, with salivary cotinine levels and the neonatal birth weight. We also compared salivary cotinine levels of infants born to mothers who were not exposed to those who were exposed to indoor smoking during their pregnancy. It was seen that salivary cotinine level increased with the exposure to SHS as noted in groups 3 and 4, as compared to groups 1 and 2 . The relationship of group 1 and group 2 was unclear. Group 1 Cotinine levels did not show values aligned with study. The higher cotinine level detected in Group 1 might be due to the fact that public smoking policy is not effectively implemented in Pakistan. Researches are also performed to assess the level of smoke in publically used areas like parks, transport and schools as these are the places where innocent victims get exposed to SHS. ${ }^{32}$

As Cotinine is not present in body normally and can only be detected due to exposure of nicotine. Another study also reported cotinine to be detected in neonates and mothers who had no exposure of 
cigarette at home hence they also infer that the exposure might be during visiting public places and they expressed it as the limitation of the study that exposure cannot be measured when visiting another house and in public areas. ${ }^{33}$

In our study indoor exposure of smoke during pregnancy was limited and evident from neonate weight but owing to traveling and recent exposure of SHS, Cotinine levels were overlapping with Group 2. This group showed least exposure to indoor SHS smoking, the similar findings were seen in another study in which cotinine was not detected in exposed neonates and it can be explained by difference in individual metabolism. ${ }^{34}$

Cotinine levels in the saliva of neonates were effectively detected in all the groups and the birth weight showed decreasing trend with increasing exposure to SHS. Except group 1, the cotinine levels increased in rest of the studied groups according to the increase in SHS exposure; $0.883 \mathrm{ng} / \mathrm{ml}, 1.153$ $\mathrm{ng} / \mathrm{ml}$ and $1.252 \mathrm{ng} / \mathrm{ml}$ in groups 2,3 and 4 respectively.

Another fact about nicotine is that it remains on the surfaces of home, in the air and in the dust of smoking members and so these things get contaminated and the other members becomes passive smokers. ${ }^{35}$

In our study the mean birth weight $(\mathrm{kg})$ in group-1 was $3.31 \pm 0.50$, in group-2 was $3.00 \pm 0.28$, in group-3 was $2.92 \pm 0.48$ and in group-4 was $2.66 \pm$ 0.41 . The birth weight showed inverse relation with the SHS exposure, however while correlating with cotinine levels only groups 2 and group 3 showed inverse relationship with birth weight that was statistically insignificant. This might be due to small sample size. Similar study to determine relationship between SHS exposure and neonate's birth weight was done in Krakow using cord blood and it shows insignificant results when comparison was made with overall cord blood cotinine and birth weight but was significant when groups were compared. ${ }^{30}$

\section{CONCLUSION}

Our study is one of the first in South Asia whereby noninvasive technique of neonate saliva was used to determine correlation between maternal exposures to SHS.Maternal exposure to SHS has inverse impact on neonate birth weight, which could have lasting effects on life of newborn baby.

\section{Limitations:}

This study was a small scale study conducted on 120 cases. The results of cotinine levels are affected by many factors such as timing, the length of time since the last exposure as well as the exposure at public places and while travelling in public transport. The outdoor exposure is the one to which people are not aware, that they are being exposed and it is difficult to quantify it.

\section{Recommendations:}

1. Large scale studies should be conducted particularly in South Asian pregnant females exposed to SHS so that more precise and comprehensive results regarding raised levels of cotinine and their inverse relationship with the birth outcome can be seen statistically.

2. Awareness of SHS hazards should be made public; especially pregnant mothers should be advised to take utmost care to avoid exposure not only to active smoking but also to SHS.

\section{REFERENCES}

1. Hecht SS, Hochalter JB, Villalta PW, Murphy SE. 2'-Hydroxylation of nicotine by cytochrome P450 2A6 and human liver microsomes: Formation of a lung carcinogen precursor. Proceedings of the National Academy of Sciences of the United States of America. 2000; 97(23):12493-12497.

2. Johnstone E, Benowitz N, Cargill A, Jacob R, Hinks L, Day I, et al. Determinants of the rate of nicotine metabolism and effects on smoking behavior. Clinical Pharmacology \& Therapeutics.2006; 80(4):319-330.

3. Kandel DB, Hu MC, Schaffran C, Udry JR, Benowitz NL. Urine nicotine metabolites and smoking behavior in a multiracial/multiethnic national sample of young adults. American Journal of Epidemiology. 2007; 165(8):901-910

4. Oscarson M. Genetic Polymorphisms in the Cytochrome P450 2A6 (CYP2A6) Gene: Implications for Inter individual Differences in Nicotine Metabolism. Drug Metabolism and Disposition. 2001; 29(2):91-95.

5. Binnie V, McHugh S, Macpherson L, Borland B, Moir K, Malik K. The validation of self reported smoking status by analysing cotinine levels in stimulated and unstimulated saliva, serum and urine. Oral Diseases. 2004;10:287-93

6. Figueiredo VC, Szklo M, Szklo AS, Benowitz N, Lozana JA, Casado L. Determinants of salivary cotinine level: a population-based study in Brazil. Rev SaudePublica. 2007;41(6):954-62

7. Nishida N, Yamamoto Y, Tanaka M, Maeda K, Kataoka K, Nakayama K et al. Association between passive smoching and salivary markers 
related to periodontitis. Journal of Clinical Periodontology. 2006; 33(10):717-23.

8. Nuca C, Amariei C, Badea V, Zaharia AG, Bucur L, Nicolae C. Salivary cotinineBiomarker of tobacco consumption in the assessment of passive smoking prevalence. Farmacia. 2012; 60(5):662-74.

9. SRNT Subcommittee on Biochemical Verification. Biochemical verification of tobacco and cessation. Nicotine and Tobacco Research. 2002; 4:149-159.

10. Etter JF, Vu Due T, Perneger TV. Saliva Cotinine Levels in Smokers and Nonsmokers. American Journal of Epidemiology. 2000; 151(3):251-8.

11. Bernert J.T. Estimation of environmental tobacco smoke exposure during pregnancy using a single question on household smokers versus serum Cotinine. Journal of exposure analysis and Environmental Epidemiology. 2002; 12:1-10.

12. Jarves M, Feyerabend C, Bryant A. Passive smoking in the home. Plasma Cotinine concentration in non-smokers with smoking partners. Tobacco Control. 2001; 10:368-374.

13. Figueiredo VC, Szklo M, Szklo AS, Benowitz N, Lozana JA, Casado L. Determinants of salivary cotinine level: a population-based study in Brazil. Rev SaudePublica.2007;41(6):954-62.

14. Hawamdeh A, Kasasbeh FA, Ahmad MA. Effects of passive smoking on children's health: a review. Eastern Mediterranean Health Journal. 2003; 9(3):441-447.

15. Melve K, Skjaerven R .Birth weight and perinatal mortality: paradoxes, social class and sibling dependencies. International Journal of Epidemiological Association 2003; 32: 625-632.

16. https://www.unicef.org/specialsession/aboutpdf/15_LowBirthweight-D7341

17. Tucker J, McGuire W. Epidemiology of preterm birth. British Medical Journal. 2004;329:675-78.

18. VS Knopik. Maternal smoking during pregnancy and child outcomes: real of spurious effect? 2009; 34(1):1-36

19. Salmasi G, Grady R, Jones J. Environmental tobacco smoke exposure and perinatal outcomes: a systematic review and metaanalyses. Acta obstetriciaetgynecologica Scandinavica. 2010; 89(4):423-41.

20. Bush PG, Mayhew TM, Abramovich DR, et al. A quantitative study on the effects of maternal smoking on placental morphology and cadmium concentration. Placenta 2000; 21:247-56.

21. Bush PG, Mayhew TM, Abramovich DR, et al. Maternal cigarette smoking and oxygen diffusion across the placenta. Placenta 2000; 21: 824-33.

22. Hofhuis W, De Jongste JC, Merkus PJ. Adverse health effects of prenatal and postnatal tobacco smoke exposure on children. Archives of disease in childhood. 2003; 88(12):1086-90.

23. Robinson JS, Moore VM, Owens JA, McMillen IC. Origins of fetal growth restriction. European Journal of Obstetrics \& Gynecology and Reproductive Biology. 2000; 92(1):13-9.

24. Eric J, Graham JB. Morphological and biological effects of maternal exposure to tobacco smoke on the feto-placental unit. Early Human Development. 2007; 83(11):699-706.

25. Hukkanen J, Gourlay SG, Kenkare S, Benowitz NL. Influence of menstrual cycle on cytochrome P450 2A6 activity and cardiovascular effects of nicotine. Clinical Pharmacology \& Therapeutics. 2005; 77(3):159-69.

26. De La Chica RA, Ribas I, Giraldo J, Egozcue J, Fuster C. Chromosomal instability in amniocytes from fetuses of mothers who smoke. Jama. 2005; 293(10):1212-22.

27. Sherman J, Young A, Sherman MP, Collazo C, Bernert JT. Prenatal smoking and alterations in newborn heart rate during transition. J ObstetGynecol Neonatal Nurs. 2002; 31(6): 680-687.

28. Sekhon HS, Keller JA, Benowitz NL, Spindel ER. Prenatal nicotine exposure alters pulmonary function in newborn rhesus monkeys. American journal of respiratory and critical care medicine. 2001; 164(6):989-94.

29. de Bernabé JV, Soriano T, Albaladejo R, Juarranz M, Calle ME, Martínez D, DomínguezRojas V. Risk factors for low birth weight: a review. Eur J ObstetGynaecolReprod Biol. 2004; 116(1):3-15.

30. Jedrychowski W, Perera F, Moroz E, Edwards S, Flak E, Bernert JT. Fetal Exposure to Secondhand Tobacco Smoke Assesed by Maternal Self-reports and Cord Blood Cotinine: Prospective Cohort Study in Karakow. Matern Child Health.2009; 13:415-23.

31. Wahabi HA, Alzeidan RA, Fayed AA, Mandil A, Al-Shaikh G, Esmaeil SA. Effects of secondhand smoke on the birth weight of term infants and the demographic profile of Saudi exposed women. BMC public health. 2013; 13(1):341.

32. Ameer S, Kandiah DM. Detection of cotinine in passive smokers exposed to environmental tobacco smoke. Biotech. 2017; 2(1):87-07

33. Sachiyo K, Kumiko A, Keiko N, Kaori K, Sonomi O. Effect of Passive Smoking Using 
Maternal and Neonatal Salivary Cotinine Measurements. Nursing research.2012;61:140-4

34. Nakajima M, Fukami T, Yamanaka H, Higashi E, Sakai H, Yoshida R, Yokoi T. Comprehensive evaluation of variability in nicotine metabolism and CYP2A6 polymorphic alleles in four ethnic populations. Clinical Pharmacology and Therapeutics. 2006;80:282-97

35. Matt GE, Quintana PJE, Hovell MF, et al. Households contaminated by environmental tobacco smoke: sources of infant exposures. Tob Cont. 2004; 13(1):29-37.

\section{The Authors:}

Dr. Wardah Anwar

Assistant Professor,

Department of Physiology,

Al-Aleem Medical College, Lahore.

Dr. Ambreen Anjum

Assistant Professor

Department of Physiology,

Al-Aleem Medical College, Lahore.

Dr. Nasreen Akhtar

Assistant Professor

Department of Forensic and Toxicology

Al-Aleem Medical College, Lahore.
Dr. Maria Anwar

Assistant Professor,

Department of Physiology,

Independent Medical College, Faisalabad

Dr. Tabinda Kazmi

Assistant Professor,

Department of Physiology,

Niazi Medical and Dental College, Sargodha.

Prof. Sibgha Zulfiqar

Head, Department of Physiology,

Shaikh Zayed Medical Complex, Lahore.

Dr. Faiza Khan

Assistant Professor

Department of Pharmacology,

Al-Aleem Medical College, Lahore.

\section{Corresponding Author:}

Dr. Wardah Anwar

Assistant Professor,

Department of Physiology,

Al-Aleem Medical College, Lahore.

E-mail: wardahtoseef@gmail.com 